\title{
High-energy astrophysical neutrinos from interactions in the Local Bubble
}

\author{
M. Bouyahiaoui ${ }^{* a}$, M. Kachelrieß ${ }^{b}$ and D. V. Semikoz ${ }^{a}$ \\ $a$
}

APC, Université Paris Diderot, CNRS/IN2P3, CEA/IRFU, Observatoire de Paris, Sorbonne

Paris Cité, 11975205 Paris, France

$b$

Institutt for fysikk, NTNU, Trondheim, Norway

E-mail: makarimeapc.in2p3.fr, Michael.Kachelriess@ntnu.no,

dmitri.semikozeapc.univ-paris7.fr

\begin{abstract}
A signal of high-energy extraterrestrial neutrinos was discovered by the IceCube experiment [1]. Neutrinos are always produced together with gamma-rays, but the gamma-ray flux from extragalactic sources cascade down to $\mathrm{GeV}$ energies due to attenuation in the intergalactic medium. Hard muon neutrino flux at $E>100 \mathrm{TeV}$ consistent with extragalactic diffuse gamma-ray background measured by Fermi LAT, however soft component of cascade neutrino flux at $E<100$ $\mathrm{TeV}$ is in conflict with it. Moreover recently a gamma-ray excess at high Galactic latitudes at energies $E>300 \mathrm{GeV}$ in the data of the Fermi telescope was reported [2]. Gamma-ray excess at $\mathrm{TeV}$ and neutrino excess at $E<100 \mathrm{TeV}$ can have common galactic origin. In this work we study possibility that both excess in IceCube astrophysical neutrino flux and gamma-ray excess at high galactic latitudes come from interactions of $\mathrm{PeV}$ cosmic rays from recent nearby source, like Vela in the walls of Local Bubble.
\end{abstract}

36th International Cosmic Ray Conference -ICRC2019-

July 24th - August 1st, 2019

Madison, WI, U.S.A.

\footnotetext{
*Speaker.
} 


\section{Introduction}

IceCube experiment detected astrophysical neutrinos in two channels. Flux of muon neutrinos from 8 years of observations [1] is consistent with power law flux of the form

$$
E^{2} F(E)=10^{-8}\left(\frac{E}{100 T e V}\right)^{2.2 \pm 0.1} \frac{\mathrm{GeV}}{\mathrm{cm}^{2} \mathrm{ssr}}
$$

Cascade flux per neutrino flavor is [1]

$$
E^{2} F(E)=(1.5 \pm 0.2) 10^{-8}\left(\frac{E}{100 T e V}\right)^{2.48 \pm 0.08} \frac{\mathrm{GeV}}{\mathrm{cm}^{2} \mathrm{ssr}}
$$

Muon neutrino flux dominate at high energies above $100 \mathrm{TeV}$, while cascade flux is higher at lower energies $E<100 \mathrm{TeV}$. High Energy Starting Events (HESE) data are consistent with both above fluxes [1] .

High energy neutrinos produced in the extragalactic sources in pion production process together with gamma-rays of similar energies. However, Universe is not transparent to gammarays. They cascade down to sub- $\mathrm{TeV}$ energies in the cosmic microwave, infrared and optical backgrounds. Diffuse Isotropic Gamma-Ray Background IGRB was measured by Fermi LAT gammaray telescope [3]. This background consistent with muon neutrino flux Eq.(1.1).

However soft component of cascade neutrino flux at $E<100 \mathrm{TeV}$ in Eq.(1.2) is too high to come from extragalactic sources, since it overproduce IGRB. It was noticed in ref. [4], that all sky Fermi gamma-ray flux is consistent with soft neutrino spectrum in Eq.(1.2). All sky gamma-ray flux is dominated by diffuse galactic emission, produced by cosmic ray interactions with gas in our Milky Way Galaxy. In ref. [5] evidence of Galactic contribution in HESE neutrino data was found based on both signal in galactic plane and at high galactic latitudes.

At $\mathrm{GeV}$ energies this emission is dominated by galactic plane contribution, but IceCube and ANTARES experiments put tight limits on contribution of Galactic plane to diffuse neutrino flux. The remaining possibility to reproduce diffuse neutrino background with Galactic contribution is either from global halo contribution [6], or from decay of Heavy Dark Matter particles [8, 9, 10] or from Local Bubble [7].

Moreover recently a gamma-ray excess at high Galactic latitudes at energies $E>300 \mathrm{GeV}$ in the Fermi LAT data was reported in ref. [2]. This excess can come from same component as diffuse neutrinos. In this work we study possibility that both gamma-ray and neutrino components are produced by cosmic ray interactions in the walls of Local Bubble.

\section{Model of local neutrino source}

In ref. [11] we proposed model, which explain cosmic ray flux at knee with major contribution of Vela pulsar. In this model we used Jansson-Farrar model for Galactic magnetic field [15] with value of turbulent field reduced to obey B/C measurements of AMS-02[16]. In anisotropic diffusion model flux of nearby young source strongly depends on the distance from Solar system to magnetic field line coming through the source of cosmic rays. If Solar system alined with this line, cosmic ray flux on Earth will be very high. In particular, in the case of Vela SN, 11000 years old, this 


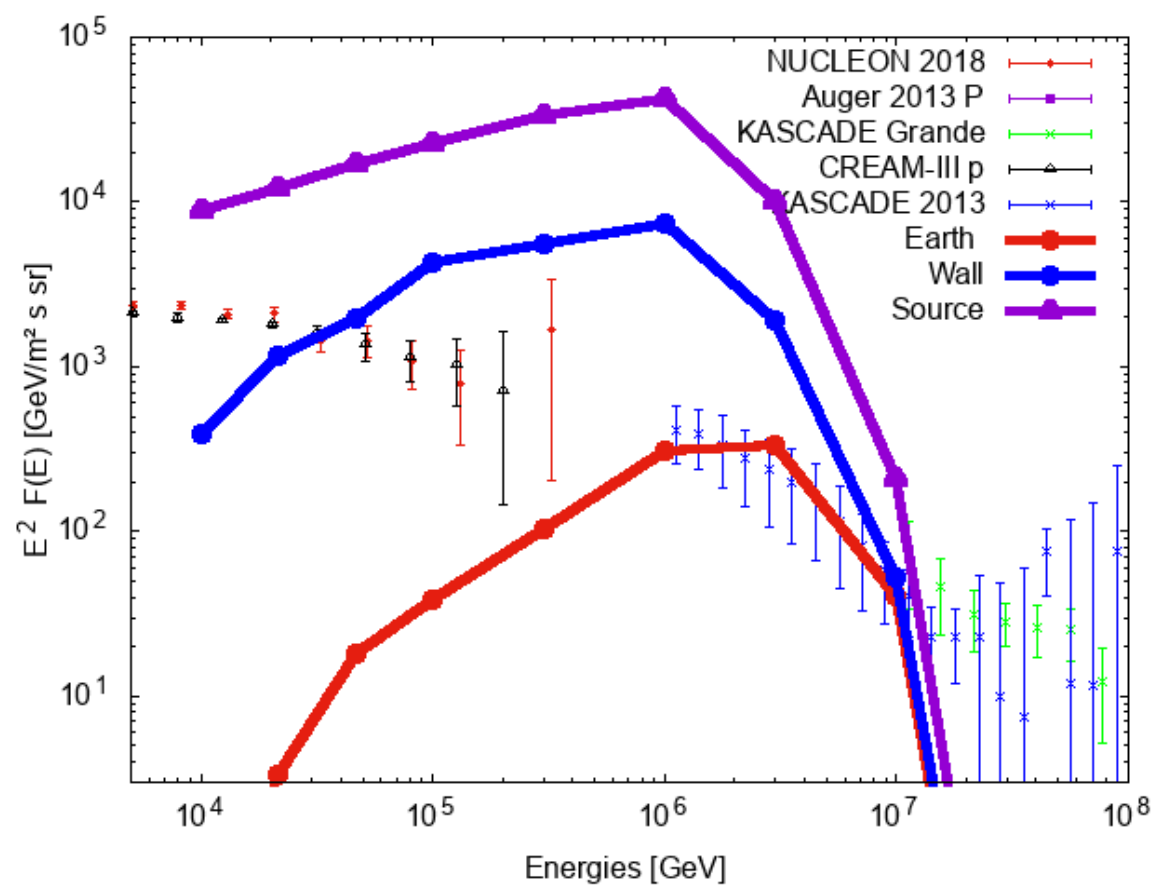

Figure 1: Contribution of Vela SN to proton flux on Earth from model of ref. [11] . Proton fluxes on source, on the Local Bubble wall and near Earth are shown with magenta blue and red lines. NUCLEON [12] and CREAM-3 [13] fluxes are direct cosmic ray measurements. KASCADE and KASCADE-Grande fluxes are indirect measurements from ref.[14].

flux is shown with magenta line in Fig. 1. Obviously, such flux violate observations on many orders of magnitude and thus Earth and Vela should not be connected directly by magnetic field line. Moreover super-bubble, a $100 \mathrm{pc}$ radius shell, in which Solar system is located at the moment, called Local Bubble serve as shield for cosmic rays coming from young nearby sources. Indeed, in ref. [11] it was shown that for typical values of magnetic field measured at walls of Local Bubble one can recover contribution of Vela SN to local cosmic ray flux with dominating contribution at knee, see red line in Fig. 1.

Cosmic ray flux on the wall of Local Bubble is shown with blue line in Fig. 1. In the 30 $\mathrm{TeV}-\mathrm{PeV}$ energy range this flux is almost two orders of magnitude higher compared to local cosmic ray flux. Also density of gas in the wall of Local Bubble is in factor 3-10 higher compared to average interstellar density. Those two factors together produce dominant contribution to the diffuse neutrino flux at $E<100 \mathrm{TeV}$. In work ref. [7] it was shown that young super nova can produce correct order of magnitude flux in the superbubble, comparable to IceCube diffuse flux at $E<100 \mathrm{TeV}$. This neutrino flux should be produced together with gamma-ray flux in cosmic ray collisions with gas in the bubble wall.

In this work we study possibility that same $\mathrm{SN}$, which produce dominant cosmic ray flux at knee give major contribution to diffuse neutrino flux measured by IceCube. In order to understand possible range of produced neutrino fluxes we studied how flux of cosmic rays in the wall depends on the properties of wall magnetic field. As parameters of our model of wall magnetic field we 
used field strength in the wall, it's width and width of transition regions between wall and magnetic field inside and our of the bubble.

The model used for the local magnetic field is very similar to the one used in [11]. We assume inside the bubble and the wall a clockwise oriented magnetic field for $y>0$ and an anticlockwise one for $y<0$. The strength of the regular magnetic field depends only on the radius for a given $z$ and is set to $B_{\text {in }}=0.1 \mu G$ inside the bubble, $B_{\text {sh }}=8-12 \mu G$ in the wall, and $B_{\text {out }}=1-3 \mu G$ outside the bubble. The Sun is assumed to be at the centre of the LB, while Vela is situated at the coordinates :

$$
r_{\text {vela }}=0.29 k p c, b_{\text {vela }}=-3.37^{\circ}, l_{\text {vela }}=263.94^{\circ} .
$$

We interpolate the transition between different magnetic field regimes by logistic functions $T(r)$, with a transition width parameter $w_{i}, i=1,2$. For the inside region $r \leq R$, we set

$$
T_{1}=\left[1+\exp \left(-\frac{r-R+w / 2}{w_{1}}\right)\right]^{-1}, T_{2}=\left[1+\exp \left(-\frac{r-R-w / 2}{w_{2}}\right)\right]^{-1}
$$

For $s=\frac{y}{|y|}$, the regular magnetic field $B_{\text {reg }}=\left(B_{\mathrm{x}}^{2}+B_{\mathrm{y}}^{2}+B_{\mathrm{z}}^{2}\right)^{1 / 2}$ is given by

$$
\begin{aligned}
& \left.B=B_{\text {in }}\left(1-T_{1}\right)+B_{\text {sh }} T_{1}\right]\left(\begin{array}{c}
s \times \sin (\theta) \\
-s \times \cos (\theta)
\end{array}\right) \exp \left(-z^{2} / z_{\text {bub }}\right)+B_{\text {out }}\left(1-\exp \left(-z^{2} / z_{\text {bub }}\right)\right), \\
& \left.B=B_{\text {sh }}\left(1-T_{2}\right)+B_{\text {out }} T_{2}\right]\left(\begin{array}{c}
s \times \sin (\theta) \\
-s \times \cos (\theta)
\end{array}\right) \exp \left(-z^{2} / z_{\text {bub }}\right)+B_{\text {out }}\left(1-\exp \left(-z^{2} / z_{\text {bub }}\right)\right),
\end{aligned}
$$

transition between wall and region outside of the bubble. We studied how flux of the walls of Local Bubble depends on parameters of magnetic field.

\section{Results}

In our model flux of cosmic rays at bubble wall is up to 100 times higher compared to flux on the Earth, see blue line in Fig. 1. Background gas in bubble walls has 3-10 times higher density compared in average interstellar medium. Together with higher flux of cosmic rays at the walls of Local Bubble this make this structure unique in the local production of secondary neutrinos and gamma-rays. In general case in ref. [7] it was shown that flux from SN located in the super-bubble is enough to explain major contribution of cascade neutrino signal in IceCube. In the ref. [2], where new diffuse gamma-ray component at 1-3 TeV energy was found in Fermi data, it was conjected that one of possible origins of that component can be contribution of nearby cosmic ray source, like Vela. Same source should give excess over extragalactic neutrino flux at energies $E<100 \mathrm{TeV}$ observed by IceCube. In this work studied this possibility in details.

We calculated neutrino and photon flux produced by cosmic rays from Vela trapped in the wall of Local Bubble. In Fig. 2 we show multi-messenger contributions to neutrino and photon fluxes calculated in our model as compared to IceCube and Fermi LAT measurements. IceCube neutrino measurements are consist of muon neutrino channel with measurements above atmospheric background at $E>100 \mathrm{TeV}$ (green band) and cascade measurements which show excess above 


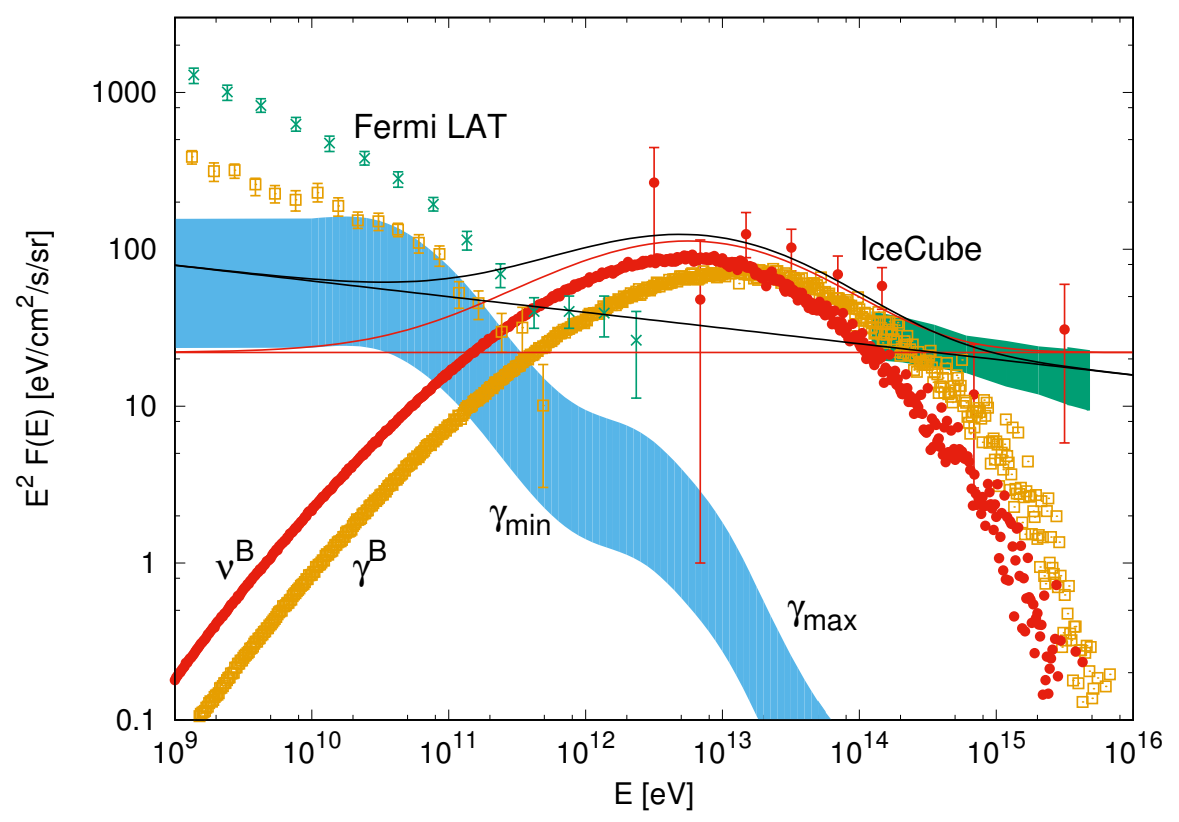

Figure 2: Multi-messenger contribution in neutrinos and gamma-rays of cosmic ray interactions in the walls of Local Bubble compired to Fermi LAT and IceCube measurements. Simulations of neutrino and gammaray fluxes from Local Bubble in our model are presented with red circles and orange squares. Neutrino flux from extragalactic sources and total neutrino flux including contribution of bubble are presented with red line for extragalactic neutrino flux $1 / E^{2}$ and with black lines for $1 / E^{2.1}$. Corresponding diffuse gamma-ray flux from extragalactic sources give contribution within blue strip normalized to diffuse gamma-ray background measured by Fermi Lat, presented with orange error-bars. Average diffuse gamma-ray flux at high galactic latitudes $|b|>20^{\circ}$ is presented with green points.

continuation of muon neutrino flux at $E<100 \mathrm{TeV}$ (red data with errorbars). Simulation of neutrino flux from Local Bubble is presented with red circles. Additionally we plot neutrino flux from extragalactic sources with red line for $1 / E^{2}$ and with black line for $1 / E^{2.1}$ and sum of both model fluxes with same line colors. One can see that combination of extragalactic fluxes with flux from Local Bubble well fit IceCube data.

Additionally we plot extragalactic diffuse gamma-ray flux measured by Fermi Lat with orange points with errorbars. We also plotted expectation of the range of contributions to gamma-ray flux from extragalactic neutrino sources. Minimal flux close to lowest values in blue band corresponds to $1 / E^{2}$ neutrino flux, while highest values normalized to measured diffuse gamma-ray background. Since up to $85 \%$ of this background come from unresolved blazars, contribution of any other sources restricted to lowest part of blue band and corresponding to $1 / E^{2}$ neutrino flux. Average diffuse gamma-ray flux at high galactic latitudes $|b|>20^{\circ}$ is presented with green points. This flux dominated by diffuse emission in local part of galaxy. In addition to expected cutoff in diffuse emission above $100 \mathrm{GeV}$ one can see new hard component at $E>300 \mathrm{GeV}$, found in ref. [2]. This component is fitted with gamma-ray flux from walls of Local Bubble.

Integral photon flux from cosmic ray interactions in the walls of Local Bubble compired to 


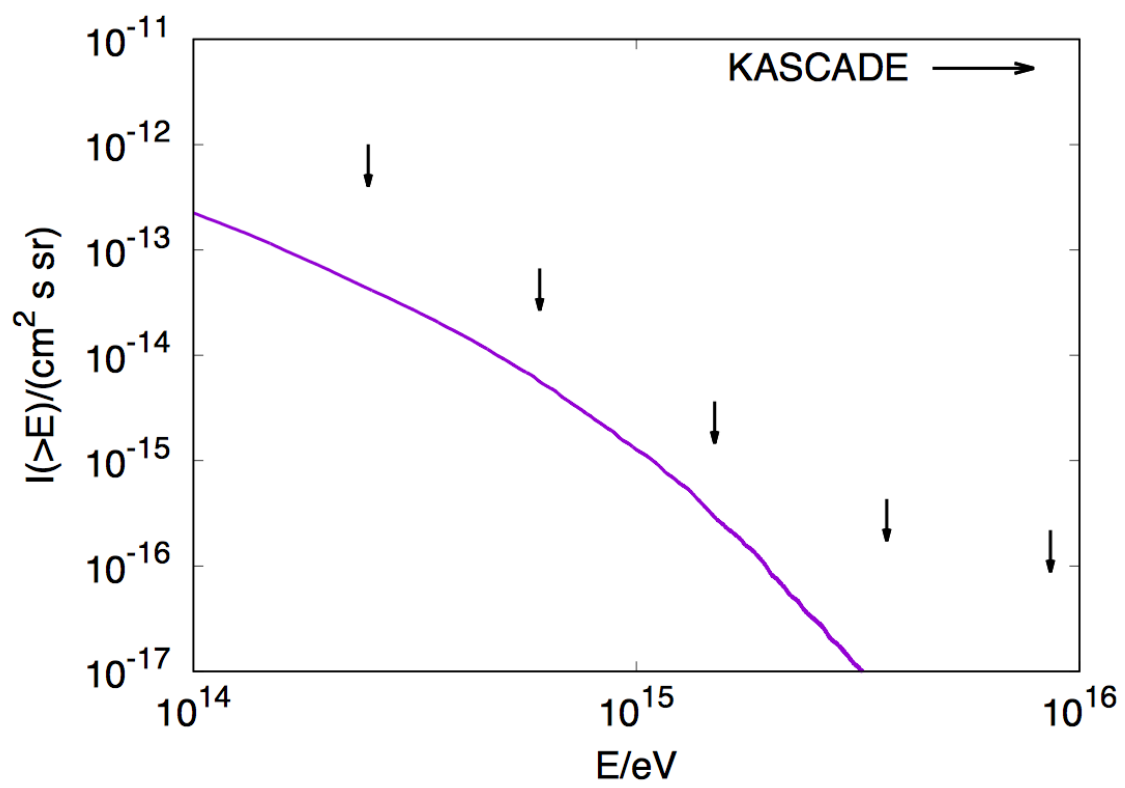

Figure 3: Integral photon flux from cosmic ray interactions in the walls of Local Bubble compired to KASCADE limits.

KASCADE limits in Fig. 3. Flux of gamma-rays at PeV energies can be measured by cosmic ray experimetns which have good hadron rejection factor, like CARPET-3 [19].

\section{Conclusions}

In this work we presented model, which explain high diffuse neutrino flux measured by IceCube at $E<100 \mathrm{TeV}$ from cosmic ray interactions in the walls of Local Bubble, structure with 100 pc radius, surrounding Solar system.

Cosmic rays at knee can be produced by young nearby SN like Vela. Local Bubble serves as magnetic shield for cosmic rays from such sources. Cosmic ray flux at Local Bubble wall is much higher compared to one measured on Earth, see Fig. 1. At the same time gas density is in 3-10 times higher in the bubble walls comparing to interstellar one. Due to both above factors significant neutrino and gamma-ray flux produced in the bubble walls, as presented in Fig. 2. Neutrino flux explain high diffuse neutrino background measured by IceCube, while photon flux explain $\mathrm{TeV}$ gamma-ray excess found recently [2]. This gamma-ray flux can be found by gamma-ray sensitive cosmic ray experiment like KASCADE, which produced upper limits factor few above our predictions, see Fig. 3. For example, Carpet-3 experiment will be sensitive in sub-PeV energy range. At lower energies one need in next generation gamma-ray experiment more sensitive compared to Fermi-LAT.

\section{References}

[1] M. G. Aartsen et al. [IceCube Collaboration], "The IceCube Neutrino Observatory - Contributions to ICRC 2017 Part II: Properties of the Atmospheric and Astrophysical Neutrino Flux," 
arXiv:1710.01191 [astro-ph.HE].

[2] A. Neronov, M. Kachelrieß and D. V. Semikoz, "Multimessenger gamma-ray counterpart of the IceCube neutrino signal,” Phys. Rev. D 98, no. 2, 023004 (2018) [arXiv:1802.09983 [astro-ph.HE]].

[3] M. Ackermann et al. [Fermi-LAT Collaboration], "The spectrum of isotropic diffuse gamma-ray emission between $100 \mathrm{MeV}$ and $820 \mathrm{GeV}$," Astrophys. J. 799, 86 (2015) [arXiv:1410.3696 [astro-ph.HE]].

[4] A. Neronov and D. Semikoz, "Neutrinos from Extra-Large Hadron Collider in the Milky Way," Astropart. Phys. 72, 32 (2016) [arXiv:1412.1690 [astro-ph.HE]].

[5] A. Neronov and D. V. Semikoz, "Evidence the Galactic contribution to the IceCube astrophysical neutrino flux,” Astropart. Phys. 75, 60 (2016) [arXiv:1509.03522 [astro-ph.HE]].

[6] A. M. Taylor, S. Gabici and F. Aharonian, "Galactic halo origin of the neutrinos detected by IceCube,” Phys. Rev. D 89, no. 10, 103003 (2014) [arXiv:1403.3206 [astro-ph.HE]].

[7] K. J. Andersen, M. Kachelrieß and D. V. Semikoz, "High-energy Neutrinos from Galactic Superbubbles,” Astrophys. J. 861, no. 2, L19 (2018) [arXiv:1712.03153 [astro-ph.HE]].

[8] V. Berezinsky, M. Kachelriess and A. Vilenkin, "Ultrahigh-energy cosmic rays without GZK cutoff," Phys. Rev. Lett. 79, 4302 (1997) [astro-ph/9708217].

[9] B. Feldstein, A. Kusenko, S. Matsumoto and T. T. Yanagida, "Neutrinos at IceCube from Heavy Decaying Dark Matter," Phys. Rev. D 88, no. 1, 015004 (2013) [arXiv:1303.7320 [hep-ph]].

[10] A. Esmaili and P. D. Serpico, JCAP 1311, 054 (2013) doi:10.1088/1475-7516/2013/11/054 [arXiv:1308.1105 [hep-ph]].

[11] M. Bouyahiaoui, M. Kachelrieß and D. V. Semikoz, "Vela as the Source of Galactic Cosmic Rays above 100 TeV,” JCAP 1901, 046 (2019) [arXiv:1812.03522 [astro-ph.HE]].

[12] N. Gorbunov et al., "Energy spectra of abundant cosmic-ray nuclei in the NUCLEON experiment," arXiv: 1809.05333 [astro-ph.IM].

[13] Y. S. Yoon et al., "Proton and Helium Spectra from the CREAM-III Flight," Astrophys. J. 839, no. 1, 5 (2017) [arXiv:1704.02512 [astro-ph.HE]].

[14] W. D. Apel et al., "KASCADE-Grande measurements of energy spectra for elemental groups of cosmic rays,” Astropart. Phys. 47, 54 (2013) [arXiv:1306.6283 [astro-ph.HE]].

[15] R. Jansson and G. R. Farrar, “'The Galactic Magnetic Field,” Astrophys. J. 761, L11 (2012) [arXiv:1210.7820 [astro-ph.GA]].

[16] M. Aguilar et al. [AMS Collaboration], "Precision Measurement of the Boron to Carbon Flux Ratio in Cosmic Rays from 1.9 GV to 2.6 TV with the Alpha Magnetic Spectrometer on the International Space Station,” Phys. Rev. Lett. 117, no. 23, 231102 (2016).

[17] A. Neronov and D. V. Semikoz, "Self-consistent model of extragalactic neutrino flux from evolving blazar population," arXiv:1811.06356 [astro-ph.HE].

[18] G. Giacinti, M. Kachelriess and D. V. Semikoz, "Reconciling cosmic ray diffusion with Galactic magnetic field models," JCAP 1807, no. 07, 051 (2018) [arXiv:1710.08205 [astro-ph.HE]].

[19] D. D. Dzhappuev et al., "Search for astrophysical PeV gamma rays from point sources with Carpet-2,” EPJ Web Conf. 207, 03004 (2019) [arXiv:1812.02663 [astro-ph.HE]]. 